\title{
Working Condition Detection of Suck Rod Pumping System via Extreme Learning Machine
}

\author{
Qian $\mathrm{Gao}^{1, \mathrm{a}}$ \\ ${ }^{1}$ School of Earth Science and \\ Resources, \\ Chang'an University, China \\ 'kinogao2010@gmail.com
}

\author{
Shaobo Sun ${ }^{2, b}$, \\ ${ }^{2}$ Department of Computer Science, \\ Resource Software College, \\ $\mathrm{Xi}$ 'an University, China \\ bunsbo@163.com
}

\author{
Jianchao Liu ${ }^{1, \mathrm{c}}$ \\ ${ }^{1}$ School of Earth Science and \\ Resources, \\ Chang'an University, China \\ 'Liuljchao@chd.edu.cn
}

\begin{abstract}
Detecting the working condition of a suck rod pumping system is an important research subject of oil extraction engineering. In the present paper, we claim a research using Extreme Learning Machine (ELM) to handle downhole dynamometer card auto recognition problems in a suck rod pumping system. First of all, we introduce a set of reasonable dynamometer card features which can reflect the characters of the cards. Then, an ELM associated with the features is constructed to recognize faults of the system automatically. The model we proposed is trained and tested by the real data acquired from Yanchang oil fields, China. Finally, we conclude based on the experiment results that ELM model has excellent generalization performance and is applicable to the automatic fault diagnosis of suck rod pumping system.
\end{abstract} ELM

Keywords: Fault Diagnosis; Suck Rod Pumping System;

\section{INTRODUCTION}

Suck rod pumping system, as the most commonly used artificial engineering system, has been well studied in many fields. Actually, Tripp [1] reported that there are nearly $90 \%$ artificially lifted wells used suck rod pumping systems, and the number in China approaches to $94 \%$ [2]. However, a suck rod pumping system is not stable when it is applied to some specific fields (e.g. oil extraction engineering in extreme environmental conditions). How to automatically recognize suck rod pumping system faults have become an urgent problem in many engineering fields.

Dynamometer card, as a plot of load vs. displacement, has become a powerful tool to analyse the downhole condition of rod-pumped wells, because different shapes of dynamometer cards represent different downhole conditions, including normal and fault conditions [3]. Therefore, recognizing the shapes of dynamometer cards can classify suck and pumping system's conditions and it is widely used in oil extraction engineering. Traditionally, suck rod pumping fault diagnosis is a process of visual interpretation and analysis of dynamometer cards by technicians [4, 5]. However, there are two significant draw backs of the traditional method. One is that the traditional method needs many experienced technicians to recognize different kinds of dynamometer cards. Even we can hire many good technicians, they may be easily influenced by objective and subjective factors, such as separated well positions inbad geography environment and complex behaviour of sucker rod systems, etc. The other one is traditional method is time-consumed to recognize faults, which cannot adapt to the modern automatic data generation and acquisition technology.

In recent decades, computer-based pattern recognition method has been developed to apply to many engineering fields successfully. Particularly, it has been suggested to utilize to improve the accuracy and efficiency of suck rod pumping system fault diagnosis [4].Actually, there are two most commonly used and popular pattern recognition methods, namely artificial neural networks (ANN) and support vector machines (SVM). On the one hand, ANN has been widely employed to handle the dynamometer card recognition problem $[2,5,6,7]$. On the other hand, SVM was proposed by Vapnik [8,9] for the purpose to construct a hyperplane or set of hyperplanes in a high and infinite dimensional space, which can be applied to solve diagnosis faults in sucker rod pumping units in many instances $[10,11$, 12].

Although, ANN and SVM have been applied to the suck rod pumping system fault diagnosis by recognizing dynamometer cards, there are still some challenging issues which cannot be overcome [13] such as:

- Slow Learning Speed: Training a SVM or ANN is relatively slow for a big data set.

- Trivial Human Intervene: In order to train a performance-well SVM or ANN, there are many parameters which have to set by human experiences.

- Poor Computational Scalability: The computational complexity of SVM and ANN is relatively high for training a model (we have to try many times).

To conquer these challenging issues, Extreme learning machine (ELM) [14], as emergent technology, has recently attracted the attention from more and more researchers. ELM works for generalized single-hidden layer feed forward networks (SLFNs). The essence of ELM is that the hidden layer of SLFNs need not be tuned. Comparing with ANN and SVM, ELM provides better generalization performance at a much faster learning speed and with least manual intervention.

In this paper, ELM is used to construct a model to achieve automation of fault diagnosis by automatically clustering dynamometer cards. The rest of the paper is organized as follows. In Section 2, we construct five 
features of dynamometer cards as inputs for an ELM. And then we give an ELM model and a fault diagnosis algorithm in the next section. Finally, the model is trained and tested by the real data acquired from Yanchang oil fields, China, to evaluate our model.

\section{FEATURE CONSTRUCTION}

The inputs of any pattern recognition algorithm are called features which can describe characteristics of object in some sense. In this section, we construct five proper features of dynamometer card for fault diagnosis. Before do that, let us introduce the standard dynamometer card in advance.

\section{A. Dynamometer Card in Suck Rod Pumping System}

Gibbs and Neely [15] indicated dynamic changes of a suck rod pumping system are influenced by a one-dimensional wave equation with viscous damping. The solution (use the Fourier coefficient method to solve) of the equation can be utilized to plot downhole dynamometer cards in a suck rod pumping unit, which can truly reflect working conditions of the subsurface pump as it may eliminate effects of the deformation, viscous resistance, vibration and inertia of the suck rod pumping system. Let's show a surface dynamometer card and calculated downhole dynamometer card which generated from Yanchang oilfield, China, in Fig. 1. We should mention that in Fig. 1 the horizontal axis is the displacement $(\mathrm{m})$ and vertical axis is the load $(\mathrm{kN})$ without direction.
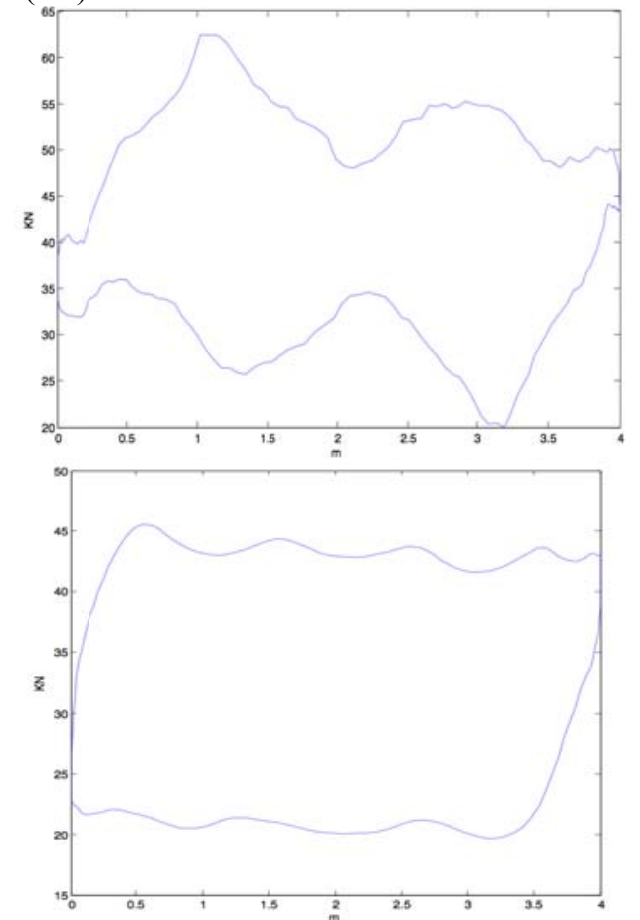

Fig.1 Surface dynamometer card(left) and calculated downhole dynamometercard(right) which are generated from Yanchang oilfield, China

\section{B. Features of Dynamometer Card}

We extract a set of geometric features for each downhole dynamometer card. The features will be the inputs of our algorithm which are essential to shape analysis and pattern recognition. General speaking, the features are centroid, perimeter and two specific areas.

Before feature extraction, dynamometer cards data need to be normalized. Because different suck rod pumping system may generate different shape dynamometer cards by displacement and load. Suppose we have a dynamometer card which is a set of samples $\left\{\left(\mathrm{X}_{\mathrm{i}}, \mathrm{Y}_{\mathrm{i}}\right)\right\}_{\mathrm{i}=1}^{1}$ Then normalization formulas are as follows:

$$
\begin{aligned}
& \bar{x}_{i}= \frac{x_{i}-x_{\min }}{x_{\text {max }}-x_{\text {min }}}, \\
& \bar{y}_{i}=\frac{y_{i}-y_{\text {min }}}{y_{\text {max }}-y_{\text {min }}}, i=1,2, \ldots . l
\end{aligned}
$$

where $x_{\text {min }}, y_{\text {min }}, x_{\text {max }}$ and $y_{\text {max }}$ are the minimal and maximal values of displacementand load respectively. Thus we can get a normalized dynamometer card as $\left\{\left(\overline{\mathcal{X}}_{i}, \overline{\mathcal{Y}}_{i}\right)\right\}_{i=1}^{\ell}$. Then the first and second features we used are the centroids of a dynamometer card. Namely

$$
\begin{gathered}
\bar{X}=\frac{\sum_{i=1}^{\ell} \bar{x}_{i}}{\ell}, \\
\bar{y}=\frac{\sum_{i=1}^{\ell} \bar{y}_{i}}{\ell} .
\end{gathered}
$$

The third feature is the perimeter of dynamometer card, namely

$$
\mathcal{P}=\sum_{t=1}^{\ell} \sqrt{\left(\bar{X}_{i+1}-\bar{X}_{i}\right)^{2}+\left(\overline{\mathcal{Y}}_{i+1}-\overline{\mathcal{Y}}_{i}\right)^{2}}
$$

where $\bar{X}_{i+1}=\bar{X}_{i}$ and $\overline{\mathcal{Y}}_{i+1}=\overline{\mathcal{Y}}_{i}$. The fourth and fifth features are two specific areas which are the red region and blue region in a dynamometer card (see Fig. 2).

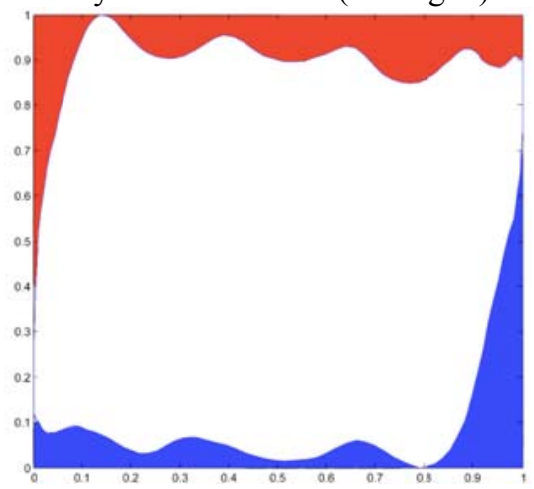

Fig.2 Normalized dynamometer card. The area of red region is the fourth feature and the area of blue region is the fifth feature.

Let us suppose that the area of red region is $\delta_{1}$ and area of blue region is $\mathcal{S}_{2}$. Assume that mis the index such that $\bar{X}_{m}=1$. Then we can compute 


$$
\begin{gathered}
\mathcal{S}_{1}=1-\frac{\sum_{i=1}^{m-1}\left(\bar{y}_{i}+\bar{y}_{i+1}\right)\left(\bar{x}_{i+1}-\bar{x}_{i}\right)}{2}, \\
\mathcal{S}_{2}=\frac{\sum_{i=\mathrm{m}}^{\ell-1}\left(\bar{y}_{i}+\bar{y}_{i+1}\right)\left(\bar{x}_{i}-\bar{x}_{i+1}\right)}{2} .
\end{gathered}
$$

Based on the five features we can construct a feature vector $f=\left(\bar{x}, \bar{y}, \mathcal{P}, \mathcal{S}_{1}, \mathcal{S}_{2}\right)^{\top}$ which is the input of our algorithm.

\section{Model CONSTRUCTION AND FAUlt Diagnosis ALGORITHM}

This section we construct an ELM model. Combining the five proper features of dynamometer cards we proposed, we introduce an algorithm for fault diagnosis of suck rod pumping systems.

We suppose that there are $\mathrm{n}$ dynamometer cards we already have. And then training samples can be obtained by construction of the five features of dynamometer cards. Assume the training sample set is $\left\{\left(f_{i}, t_{i}\right)\right\}_{i=1}^{n} \subset \mathbb{R}^{5} \times \mathbb{R}^{d}$ Here $d$ means we consider $d$ different fault patterns. The ELM model with $\mathcal{N}$ hidden nodes and active function $g(f)$ are mathematically modeled as following:

$$
\sum_{j=1}^{N} \beta_{j} g\left(f_{i}\right)=\sum_{j=1}^{N} \beta_{j} g\left(w_{j}^{\top} f_{i}+b_{j}\right)=\mathcal{O}_{i}, i=1,2, \ldots, n,
$$

where $\mathcal{W}_{j}=\left(\mathcal{W}_{j 1}, \mathcal{W}_{j 2}, \mathcal{W}_{j 3}, \mathcal{W}_{j 4}, \mathcal{W}_{j 5}\right)^{\top}$ is the weight vector connecting the $j^{\text {th }}$ hidden nodeand the input nodes, $\beta_{j}=\left(\beta_{j 1}, \beta_{j 2}, \ldots, \beta_{j d}\right)^{\top}$ is the weight vector connecting the $j^{t h}$ hidden node and the output nodes, and $b_{j}$ is the threshold of the $j^{t h}$ hidden node. The outputnodes are chosen linear in this paper. The ELM model with $\mathcal{N}$ hidden nodes with activefunction $g(f)$ can approximate the $n$ samples with minimizing error means

$$
\sum_{i=1}^{n}\left\|t_{i}-\sigma_{i}\right\|^{2} \text {. }
$$

The above $\mathrm{n}$ equations can be written by a compact matrix form like $\mathcal{H} \beta=\mathcal{T}$, Where $\mathcal{H}=\left(g\left(w_{j}^{\top} \mathfrak{f}_{i}+\right.\right.$ $\left.\left.b_{j}\right)\right)_{n \times \mathcal{N}}, \beta=\left(\beta_{1}^{\top}, \ldots, \beta_{\mathcal{N}}^{\top}\right)_{\mathcal{N} \times 5}^{\top}$, and $\mathcal{T}=\left(t_{1}^{\top}, \ldots, t_{n}^{\top}\right)_{n \times 5}^{\top}$. As named in Huang et al. [14], $\mathcal{H}$ is called the hidden layer output matrix of the neuralnetwork. Thus, we can get an estimation of $\beta$ as

$$
\widehat{\beta}=\mathcal{H}^{+} \mathcal{T}
$$

where $\mathcal{H}^{+}$is the Moore-Penrose generalized inverse of matrix $\mathcal{H}$. The crucial point ofELM model is how to choose the parameters $w$ and $b$. Huang et al. [14] suggested that $w$ and $b$ can be assigned randomly. For example, they can be assigned from a uniformdistribution. They also construct a theoretical point to justify the random strategy can keep the generalization performance of ELM [13].

So far, combining the ELM model we described and the features of dynamometer cards we proposed in the previous section, we can suggest a following fault diagnosis algorithm:

\section{Algorithm 1 Fault Diagnosis Algorithm via ELM Input:}

$n$ dynamometer cards for training.

\section{Output:}

Estimation output weight $\hat{\beta}$

1: Construct a training sample set $\mathcal{S}=\left\{\left(f_{i}, t_{i}\right)\right\}_{i=1}^{n}$ based on (2), (3) and (4).

2: Randomly assign input weight $\mathrm{w}$ and bias $b$.

3: Calculate the hidden layer output matrix $\mathcal{H}$.

4: Calculate the output weight $\beta$ as

$$
\widehat{\beta}=\mathcal{H}^{+} \mathcal{T}
$$

Where $\mathcal{T}=\left(t_{1}^{\top}, \ldots, t_{n}^{\top}\right)^{\top}$

Finally, if we have a test sample $f_{\text {test }}$, then we can estimate a label of the test sample as

$$
t_{\text {test }}=\operatorname{sign}\left(\sum_{j=1}^{\mathcal{N}} \hat{\beta}_{j} g\left(w_{j}^{\top} f_{\text {test }}+b_{j}\right)\right) \text {. }
$$

\section{EXPERIMENT STUDY}

In this section, the performance of the proposed fault diagnosis algorithm is compared with the popular algorithms of feed forward neural networks like the conventional BP algorithm and support vector machines (SVMs) on a set of dynamometer card data which is generated from Yanchang oil field, China.

As we known, there are almost 9 typical faults in a suck rod pumping system which are stands for gas influence, feed liquid failure, sucker rod breakage, oil of high viscosity, travelling valve leakage, pump bumping in bottom dead position, pump bumping in top dead position, standing valve leakage and sand production. However, the faults of feed liquid failure and sucker rod breakage are the most common faults in Yanchang oil field and the others are less found. Therefore, we select a dynamometer card data set which contains 330 samples. And the samples include 3 types, namely normal working of the pumping unit, 130 samples; feed liquid failure, 100 samples; sucker rod breakage, 100 samples. We use 90 normal samples, 70 feed liquid failure samples and 70 sucker rod breakage samples as a training sample set. And the other samples are the testing set.

For the three competitive algorithms, we use the existed packages to demonstrate the performance. For BP we use the MATLAB code. For SVMs, the study is carried out by a LIBSVM package which can be found the MATLAB version at [16]. The same to standard ELM, the MATLAB version code can be found at [17].

There are several settings for the three algorithms. First, we use the radial basis function (RBF) $\exp \left(-\frac{\|x-a\|^{2}}{b}\right)$ as the active function in BP, ELM and SVM. Then, thenumber of hidden nodes is gradually increased by an interval of 5 and the nearly optimal number of nodes for BP is selected based on 5 folder cross-validation method. However, we fix the 
hidden nodes of ELM as 20 and randomly generated $w$ and $b$ 20times. Then we select the optimal parameters via the best prediction error. For SVM,we estimate the prediction error using different combination of cost parameters $\mathcal{C}$ and kernel parameters $\gamma$ : $\mathcal{C}=\left[2^{12}, 2^{11}, \ldots, 2^{-1}, 2^{-2}\right]$, and $\gamma=\left[2^{4}, 2^{3}, \ldots, 2^{-9}, 2^{-10}\right]$. Therefore, for each problem we try $15 \times 15=225$ combinations. Then we choose the best combination via 5 folder cross-validation method. Then classification results of the three algorithms are shown in Table 1.

TABLE 1 THE RESULTS OF BP, SVM AND ELM ON DYNAMOMETER CARD DATA SET.

\begin{tabular}{|cccc|}
\hline Algorithm & $\begin{array}{c}\text { TrainingTime } \\
\text { (second) }\end{array}$ & $\begin{array}{c}\text { TestingTime } \\
\text { (second) }\end{array}$ & Accuracy \\
\hline BP & 108.2 & 11.5 & $78 \%$ \\
\hline SVM & 303.1 & 3.3 & $83 \%$ \\
\hline ELM & 28.7 & 5.6 & $89 \%$ \\
\hline
\end{tabular}

Let us make some comments for the results. From Table 1 we can observe that ELM algorithm has the least training time. We think the reason is that all the parameters in ELM algorithm are randomly selected, so we do not need to use any extra strategy(e.g., cross-validation) to find them. This dramatically reduces the training time. For the testing time, we can find that SVM algorithm has he fast speed. That is because it is well known that SVM has a special ability to select typical samples in training set as so-called support vectors as less as possible. Thus, the prediction time is much less than any other algorithm due to the sparse support vectors. The table also has shown that ELM algorithm has the best prediction ability (generalization ability) due to the highest accuracy for classifying the different type faults.

To sum up, ELM algorithm associated with the proper five features we proposed is not only training the model fast which due to the less human interaction, but also the classification accuracy is good for the dynamometer card data set of Yanchang oil field,China.

\section{CONCLUSION}

In this article, we applied ELM successfully to classify downhole dynamometer card of suck rod pumping system which is generated in Yanchang oil field, China. Firstly, we proposed five proper features of downhole dynamometer card of suck rod pumping system. And then we introduced the ELM. Combining the feature construction strategy and ELM, we constructed an efficient fault diagnosis algorithm, that is Algorithm 1.Based on the experiment study, we found the ELM associated the new five features of dynamometer cards is a more stable model with higher diagnosis accuracy, better generalization capability and lower computational complexity comparing with the conventional approaches (BP neural network and SVM). Our study in Yanchang oil field, China, proved that ELM is more competitive than BP and SVM for fault diagnosis of suck rod pumping system and could help engineers to interpret downhole condition of oil wells promptly and correctly.

\section{REFERENCES}

[1] H.A.Tripp: A review: analyzing beam-pumped wells. Journal of Petroleum Technology, Vol. 41(1989), p. 457-458.

[2] F.Ding, andZ.Dixun: Neural network expert system for rod pumping diagnosis. China Petroleum Machinery, Vol 30(1996), p. 1-4.

[3] J.D.Rogers,C.G.Guey and W.B.Oldham: Artificial neural networks for identification of beam pump dynamometer load cards. In SPE Annual Technical Conference and Exhibition, SPE Press: USA(1990).

[4] R.Dickinson andJ.Jennings: Use of pattern-recognition techniques inanalyzing downhole dynamometer cards. SPE Production Engineering, Vol 5(1990), p. 187-192.

[5] X. Peng, X.Shijin andY. Hongwei: Application of self-organizing competitive neural network in fault diagnosis of suck rod pumping system. Journal of Petroleum Science and Engineering, Vol 58(2007), p. 43-48.

[6] X. Peng, X.Shijin andY. Hongwe and Z. Huiqun: Application of self-organizing competitive neural network to fault diagnosis of sucker rod pumping system in Jiangsu oilfield. Geological Journal of China Universities, Vol 2(2006), p. 1-14.

[7] M.D. Bezerra,L.Schnitman, M.Filho,A.M. Jose,and F.Souza: Pattern recognition for downhole dynamometer card in oil rod pump system using artificial neural networks. In ICEIS, Elsevier: USA(2009).

[8] N.Vapnik: Statistical learning theory. Wiley: NY(1998).

[9] C.Corinna, and V.Vapnik, Support-vector networks. Machine learning,20(1995), p. 273-297.

[10] T. Jingwen,G. Meijuan,L. Yanxia,Z. Hao,and L.Kai: The fault diagnosis system with self-repair function for screw oil pump based on support vector machine. IEEE International Conference on Robotics and Biomimetics, IEEE: USA(2007).

[11] T.Jingwen,G. Meijuan,L. Kai,and Z. Hao: Fault detection of oil pump-based on classify support vector machine. IEEE International Conference on Control and Automation, IEEE: USA(2007).

[12] L. Kun, G. Xianwen,T. Zhongdaand Q.Zhixue: Using the curve moment and the pso-svm method to diagnose downhole conditions of a sucker rod pumping unit. Petroleum Science, Vol 10(2013), p. 73-80.

[13] H. Guangbin,W. Dianhui andL. Yuan: Extreme learning machines: a survey. International Journal of Machine Learning and Cybernetics, Vol 2(2011), p.107-122.

[14] H. Guangbin, Z. Qinyu and S.Cheekheong: Extreme learning machine: theory and applications. Neuro computing, Vol 70(2006), p. 489-501.

[15] S.G. Gibbs and A.B.Neely: Computer diagnosis of downhole conditions in sucker rod pumping wells. Journal of Petroleum Technology, Vol 18(1966), p. 91-98.

[16] Information on http://www.csie.ntu.edu.tw/ cjlin/libsvm/.

[17] Information on http://www.ntu.edu.sg/home/egbhuang/. 